\title{
The Status of Pulsar Emission Theory
}

\author{
D.B. Melrose
}

School of Physics, University of Sydney, NSW 2006, AUSTRALIA

\begin{abstract}
It is argued that there is now a preferred pulsar radio emission mechanism, involving beam-driven Langmuir turbulence. A testable prediction is that, at least in a statistical sense, features in the spectra of pulsars should scale with the plasma frequency, $\nu_{\mathrm{GJ}}$, implied by the Goldreich-Julian number density.
\end{abstract}

\section{Introduction}

The identification of the pulsar radio emission mechanism is one of the longeststanding unresolved problems of pulsar physics. Recent progress on radio emission theory, which is well represented by papers presented at this meeting, suggests that current opinions are tending toward a consensus concerning the emission mechanism. In section 2 the arguments pointing toward this specific emission mechanism are summarized. The emission mechanism itself is described in section 3. There remain many outstanding difficulties one of which is discussed in section 4. A more personal overview of the present status of pulsar emission theory is presented in section 5 .

\section{Identification of the Emission Mechanism}

There are statistical arguments in favor of pulsar radio emission originating at modest heights along dipole-like magnetic field lines near the last closed field line emerging from the polar-cap regions of the neutron star. The evidence in favor of emission from dipole-like field lines originating from the polar cap includes the sweep of the linear polarization and the variation of the pulse width with period $P$. The sweep of the linear polarization is consistent with the rotating vector model (Radhakrishnan and Cooke 1969), modified by jumps between orthogonal modes in a broad class of pulsars (Stinebring et al. 1984). The statistical dependence (Rankin 1990), $W \propto 1 / P^{1 / 2} \sin \alpha$, of the pulse width on $P$ and $\alpha$, which is the angle between the rotation and the dipole axes, suggests a source region located near the last closed dipolar field line. Statistical evidence in favor of a radius-to-frequency mapping (Blaskiewicz, Cordes and Wasserman 1991) suggests an emission height of $r / R_{*} \sim 10-100$, where $R_{*}$ is the radius of the star.

These features indicate that the emission originates in the outflowing pair plasma well above the surface of the neutron star. Pulsar radio emission is extremely bright, requiring a coherent emission process, which should involve 
some form of plasma instability (Melrose 1995, Lyutikov 1998), although which specific instability is uncertain. Relevant instabilities may be classified according to the nature of the wave-particle interaction (Landau resonance, cyclotron resonance, curvature emission, free electron maser emission), the source of free energy (beams, distributions with $\partial f(\gamma) / d \gamma>0$, anisotropy) and the mode (Langmuir, Alfvén, magnetoacoustic) of the waves that grow in the instability. Growth of beam-driven Langmuir waves is the most widely favored instability.

The radio emission from millisecond pulsars is closely analogous to that from ordinary pulsars, suggesting that the emission mechanism is not sensitive to the differences between the two classes of pulsars. The similarity of emission from ordinary and millisecond pulsars strongly favors a beam-driven instability generating Langmuir waves. This follows from the inference that the emission can be similar in these two cases only if it is insensitive to parameters that are markedly different in the two classes of pulsars. This rules out cyclotron effects, which depend explicitly on $B$, and also suggests that the emission mechanism is insensitive to the Alfvén speed, $v_{A} \propto B$. One parameter does have a relatively small spread: the Goldreich-Julian number density, $n_{\mathrm{GJ}} \propto B / P$, is roughly the same for ordinary and millisecond pulsars. This suggests that a plausible natural frequency that involves $B$, but is not strongly sensitive to it, is the plasma frequency defined by $n_{\mathrm{GJ}}$ :

$$
\nu_{\mathrm{GJ}}=\left(\frac{e^{2} n_{\mathrm{GJ}}}{4 \pi^{2} \varepsilon_{0} m}\right)^{1 / 2}=7.5 \mathrm{GHz}\left(\frac{B_{*}}{10^{12} \mathrm{G}}\right)^{1 / 2}\left(\frac{P}{100 \mathrm{~ms}}\right)^{-1 / 2}\left(\frac{r}{R_{*}}\right)^{-3 / 2},
$$

where $B=B_{*}\left(r / R_{*}\right)^{-3}$ is assumed, with $B_{*}$ the polar magnetic field.

\section{Relativistic Plasma Emission}

The foregoing arguments favor an emission mechanism involving a plasma-beam instability that produces Langmuir waves. This emission mechanism is referred to here as relativistic plasma emission, based on an analogy with plasma emission from the solar corona (Melrose 1991). The wave properties in a pulsar plasma are quite different from those in a nonrelativistic plasma, and an essential ingredient in identifying the emission mechanism is the identification of the properties of the relevant waves. The pulsar plasma above the polar caps is assumed to be generated by a pair cascade (Daugherty and Harding 1983), which results in a pair number density $n=M n_{\mathrm{GJ}}$, with multiplicity $M \sim 10^{2}-10^{4}$, a bulk Lorentz factor $\gamma_{b}=\left(1-v_{b}^{2} / c^{2}\right)^{-1 / 2} \sim 10^{2}-10^{3}$, and a large spread in Lorentz factors $\langle\gamma\rangle \gg 1$ in its rest frame. The particles are assumed to radiate away all their perpendicular (to $\mathbf{B}$ ) energy so that the plasma is one dimensional.

The low-frequency (well below the cyclotron frequency) wave modes in the rest frame of the pulsar plasma are illustrated in Figure 1(a) (Lominadze, Mikhailovskii and Sagdeev 1979; Arons and Barnard 1986). There are three wave modes, and only two are shown. For parallel propagation (wave angle $\theta=0$ between $\mathbf{k}$ and $\mathbf{B}$ ) these are the Alfvén (A) and Langmuir (L) modes. An important point in the following discussion arises from the fact that these two dispersion curves intersect, at what is referred to as the cross-over frequency, $\sim \omega_{p}\langle\gamma\rangle^{1 / 2}$. The parallel Langmuir mode extends from a cutoff $\left(z=\omega / k_{\|}=\infty\right)$ 


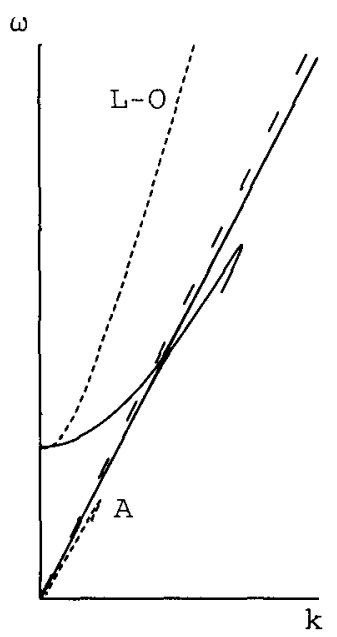

(a)

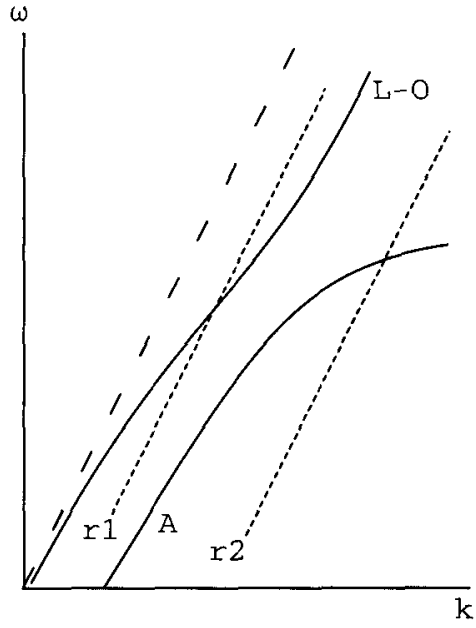

(b)

Figure 1. (a) The dispersion curves for the Alfvén (A) and L-O modes in a pulsar plasma: the solid line is for nearly parallel propagation, the dotted line for highly oblique propagation, and the dashed line is the light line $\omega=k_{\|}$. (b) On a different scale for nearly parallel propagation the region near the cross-over between the Alfvén and Langmuir (L) modes is indicated, with $\mathrm{r} 1$ and $\mathrm{r} 2$ denoting resonance lines with $v_{b}>z_{A}$ and $v_{b}<z_{A}$, respectively.

at the relativistic plasma frequency, $\omega_{p}\left\langle\gamma^{-3}\right\rangle^{1 / 2} \sim \omega_{p} /\langle\gamma\rangle^{1 / 2}$, to a maximum frequency just above the frequency at which the dispersion curve crosses the light line $(z=c)$, which frequencies are just above and just below the crossover frequency, respectively. [Note that one has $\left\langle\gamma^{-n}\right\rangle \sim 1 /\langle\gamma\rangle$; explicit expressions for a one-dimensional relativistic thermal distribution were given by Melrose and Gedalin (1999).] The parallel Alfvén mode has dispersion relation $z=z_{A}=v_{A} /\left(1+v_{A}^{2} / c^{2}\right)^{1 / 2}$, where the Alfvén speed $v_{A}=B /\left(\mu_{0} n m\langle\gamma\rangle\right)^{1 / 2}$ is typically $\gg c$ in a pulsar plasma. The significance of the cross-over frequency is that for oblique propagation the dispersion curves reconnect, both deviating away from the cross-over point. For oblique propagation $(\theta \neq 0)$ the lower portion of the Langmuir mode and the upper portion of the parallel Alfvén mode reconnect to form the $\mathrm{L}-\mathrm{O}$ mode. The lower portion of the parallel Alfven mode reconnects with the portion of the parallel Langmuir mode near the maximum frequency, which now occurs in the oblique Alfvén mode.

The third mode, not shown, called variously the magnetoacoustic mode, the $\mathrm{X}$ mode and the $t$ mode, is degenerate with the parallel Alfvén mode for $\theta=0$ and is only weakly dependent on angle of propagation. Escaping radiation must be in one of the two modes, the L-O or X modes, that extend to arbitrarily high frequencies.

A beam instability involves one component of the plasma flowing relative to another component. The most effective form of beam instability in a pulsar 
magnetosphere is that suggested by Usov (1987): it is assumed that the pair cascade occurs in a nonstationary manner producing initially distinct clouds of pairs, and the counterstreaming motion arises when the slower particles in a preceding cloud are over taken by the faster particles in a following cloud (Asseo and Melikidze 1998). The resonance condition for a beam instability, when a lower density fast beam passes through a higher density, non-streaming background plasma, is that the beam speed equal the phase speed of the waves in the background plasma. Two examples for resonance conditions are illustrated schematically in Figure 1(b): r1 corresponds to $v_{b}>z_{A}\left(\gamma_{b}>v_{A}\right)$ and it intersects the nearly-parallel L-O mode above the cross-over frequency; $\mathrm{r} 2$ corresponds to $v_{b}<z_{A}\left(\gamma_{b}<v_{A}\right)$ and it intersects the Alfvén mode below the cross-over frequency. For $v_{b} \approx z_{A}\left(\gamma_{b} \approx v_{A} / c\right)$ resonance can occur with the Alfvén mode over a broad range of frequencies well below the cross-over frequency.

The only characteristic frequencies that appear in these dispersion curves are the cutoff frequency for the Langmuir waves and the cross-over frequency. Both are proportional to $\nu_{\mathrm{GJ}}$ for $n \propto n_{\mathrm{GJ}}$. However, a beam instability can involve only waves with $z<1$, and hence Langmuir waves near their cutoff frequency (corresponding to $z=\infty$ ) cannot be generated directly. It follows that the only plausible resonance involves waves near the cross-over frequency. Whether these are called Langmuir waves or Alfvén waves is perhaps a semantic point, and once the semantic differences are discounted, there is an increasing trend in the literature to concentrate on a beam instability that produces such waves as the first step in the pulsar emission process.

For ordinary pulsars the resonance condition $\gamma_{b} \approx v_{A} / c$ could be satisfied well inside the light cylinder only for the extremely energetic primary particles (e.g., with $\gamma_{b} \sim 10^{7}$ ), and then, due to the decrease in $v_{A}$ with $r$, the instability would be restricted to one point along a given field line. For the favored instability (Usov 1987) only Alfvén waves near their maximum frequency can be generated, and these waves cannot escape directly. It follows that a second stage in the emission process is required to convert part of the energy in the waves generated through the instability into escaping radiation (in either the $\mathrm{L}-\mathrm{O}$ or $\mathrm{X}$ modes), as is the case for plasma emission in the solar corona (Melrose 1991). There is no consensus on the plasma processes involved in this second stage.

\section{Difficulties with Relativistic Plasma Emission}

There are several difficulties with this favored emission mechanism, with perhaps the most serious being the implied frequency of emission. The foregoing discussion implies that the waves generated in the instability have frequency $\omega_{p}\langle\gamma\rangle^{1 / 2}$ in the rest frame of the plasma. Several further assumptions are needed to estimate the frequency of emission. The simplest assumptions are that the escaping radiation is produced in the rest frame of the plasma with no substantial change in frequency from $\omega \sim \omega_{p}\langle\gamma\rangle^{1 / 2}$, and that the resulting waves are propagating nearly along the field lines with phase speed close to $c$. Then one can make a Lorentz transformation to the pulsar frame, taking care to redefine $\omega_{p}$ in terms of the number density in this new frame. (The density $n_{\mathrm{GJ}}$ applies in the pulsar 
frame.) This leads to a frequency

$$
\nu \sim \nu_{\mathrm{GJ}}\left(2 M \gamma_{b}\langle\gamma\rangle\right)^{1 / 2}
$$

with $\nu_{\mathrm{GJ}}$ given by (1).

To predict ths frequency of observation requires estimates of $\left(2 M \gamma_{b}\langle\gamma\rangle\right.$ in (2) and of $r / R_{*}$. A seemingly plausible estimate is that the energy density of the secondary pairs is comparable to that in the primary particles. With the estimated Lorentz factor $\sim 10^{7}$ for the primary particles, this implies $M \gamma_{b} \sim$ $10^{7}$. The lowest frequency would come from the greatest height, and adopting $r / R_{*} \sim 10^{2}$. Adopting these values, with $\langle\gamma\rangle \sim 10,(2)$ with (1) gives a minimum frequency $\nu \sim 100 \mathrm{GHz}$, far too high (Kunzl et al. 1998, Melrose and Gedalin 1999) to be compatible with observations, with $\nu \sim 100 \mathrm{MHz}$ being observed from many pulsars. The lowest values that might be considered plausible in the context of polar cap models are $M \sim 10^{3}, \gamma \sim 10^{2},\langle\gamma\rangle \sim 10$, which with the other choices of parameters above gives a minimum frequency of order $10 \mathrm{GHz}$, which is still far too high. Only with the most extreme estimates that would minimize the frequency (2) could one account for the observed lowest frequency. For example, $M \sim 1$, corresponding to secondary pairs of comparable density to the primary particles, $\gamma \sim 10^{2},\langle\gamma\rangle \sim 10$ and $r / R_{*} \sim 10^{3}$ would allow the lowest frequencies to be consistent with this emission mechanism.

\section{Towards an Acceptable Model for the Emission Mechanism}

The arguments in favor of relativistic plasma emission over various alternatives are strong, especially when one requires that the emission from both ordinary and millisecond pulsars be due to the same mechanism. If one assumes that this is the correct emission mechanism, then one may ask what other assumptions need to be made to account for the various observed features of pulsar radio emission. The analogy with plasma emission from the solar corona may provide some helpful indications on what other assumptions may be required. One specific suggestion is discussed here: the plasma in the source region may be highly inhomogeneous.

The suggestion that the production of pairs is inhomogeneous and nonstationary has long been considered plausible (Ruderman and Sutherland 1975). In the superstrong magnetic field, once a pair cascade is triggered its lateral extent is limited by the distance photons travel across the field before decaying into pairs. As a result one would expect strongly field aligned inhomogeneous structures, that is, plasma columns above the polar cap regions. Such structures have been invoked for several different reasons by others at this meeting (Kuz'min, these proceedings; Rankin and Deshpande, these proceedings; Lyubarskii and Petrova, these proceedings). There is indirect evidence for such structures in the solar corona: strong outward guiding of radio bursts along ducts is required to account for the apparent heights of type II and type III sources (Duncan 1979), highly collimated structures appear to be required to account for the directivity of type I sources (Bougeret and Steinberg 1977), and reflection at the sharp plasma gradients also seems to be required to account for depolarization of some radio bursts. By analogy, assuming that the pair plasma is confined to columns in a pulsar magnetosphere can overcome several difficulties with the 
current theory. First, the difficulty discussed above concerning the frequency of emission by postulating emission from the low-density edges of the columns into the underdense regions, with $M \sim 1$, between the columns. Second, reflection from the edges of the columns can lead to a mixture of the two escaping modes, as is required to account for the flips between orthogonal modes (Stinebring et al. 1984). Third, the model allows there to be relatively few pairs (von Hoensbroech, Lesch and Kunzl 1998) in localized source regions.

In conclusion, one can have some optimism about the present status of the pulsar radio emission theory. There is a growing consensus on the general nature of the emission mechanism, albeit with diverse attitudes to some of the important details. It is important that specific models be worked out in more detail and be tested against observations. For example, a specific idea introduced above is that the frequency of pulsar emission should be determined by the frequency $\nu_{\mathrm{GJ}}$, cf. (1), implying that at least in a statistical sense the frequency spectra of pulsars should exhibit common features when plotted as a function of $\nu / \nu_{\mathrm{GJ}}$.

\section{References}

Arons, J., and Barnard, J.J. 1986, ApJ, 302, 120

Asseo, E., and Melikidze, G.I. 1998, MNRAS, 301, 59

Blaskiewicz, M., Cordes, J.M., and Wasserman, I. 1991, ApJ, 370, 643

Bougeret, J.-L., and Steinberg, J.-L. 1977, Solar Phys., 61, 777

Daugherty, J.K., and Harding, A.K. 1983, ApJ, 273, 761

Duncan, R.A. 1979, Solar Phys., 63, 398

von Hoensbroech, A., Lesch, H., and Kunzl, T. 1998, A\&A, 336, 209

Kunzl, T., Lesch, H., Jessner, A., and von Hoensbroech, A. 1998, ApJ, 505, L139

Lominadze, D.G., Mikhailovskii, A.B., and Sagdeev, R.Z. 1979, Sov. Phys. JETP, 50, 927

Lyutikov, M. 1998, MNRAS, 293, 447

Machabeli, G.Z., Usov, V.V. 1979, Sov. Astron. Lett., 5, 238

Melrose, D.B. 1991, ARA\&A, 29, 31

Melrose, D.B. 1995, J. Astrophys. Astr., 16, 137

Melrose, D.B., and Gedalin, M. 1999, ApJ, 521, 351

Radhakrishnan, V., and Cooke, D.J. 1969, Astrophys. Lett., 3, 225

Rankin, J.M. 1990, ApJ, 352, 247

Ruderman, M.A., and Sutherland, P.G. 1975, ApJ, 196, 51

Stinebring, D.R., et al. 1984, ApJS, 55, 247

Usov, V.V. 1987, ApJ, 320, 333 